\title{
Optimization of accelerated testing through design of experiment for ageing of lead-free electronic interconnection material
}

\author{
M. Catelani ${ }^{1}$, V.L. Scarano ${ }^{1, \star}$, and R. Berni ${ }^{2}$ \\ 1 University of Florence, Department of Electronics and Telecommunications, Via S. Marta 3, 50139 Florence, Italy \\ 2 University of Florence, Department of Statistics, Viale Morgagni 59, 50134 Florence, Italy
}

Received: 23 October 2012 / Accepted: 22 November 2012

\begin{abstract}
Understanding the ageing of materials for electronic application is a complex challenge, in particular for composite material as electrically conductive adhesive consisting of a nonconductive polymer binder and conductive filler particles. This research involves a large amount of parameters related to both operating conditions and material structure, which act together. Accelerated testing, with the aim to consume rapidly lifetime without inducing incorrect failure mechanisms, is difficult to optimize and its modelling to describe the ageing process is a challenge. To reach this aim could be interesting for the characterization of the material but above all for the general validity of the proposed methodology.
\end{abstract}

Keywords: Accelerated life testing; ageing; electrically conductive adhesive; experimental design; lifetime data

\section{Introduction}

When new materials are used in electronic devices, it is necessary to verify their behaviour in defined conditions. In several applications such as military, aerospace, telecommunication, medical implants, automotive it is also fundamental to guarantee a high reliability levels. In particular, the assessment of the reliability of a new material is a non-trivial challenge [1], because of the new structure of the material and the absence of field data [2].

In this research activity we focused on material as Electrically Conductive Adhesive (ECA). ECA is constituted by polymers that represent the adhesive part, named matrix. These polymers often are epoxy resins. The choice is due to their stability, adhesion strength and great resistance, both mechanical and chemical. Moreover this material adheres to most of surfaces, both metallic and plastic. The other part that made up the ECA is the filler. The filler is metallic material that is drowned in the epoxy resin matrix and it is useful to let electrically conductive the adhesive. The metallic particles that made up the filler are diffused within the matrix in an absolutely random way.

These adhesives that can be used in electronic devices as electrically conductive materials and packaging materials, would have a great future in electronics if they will be also demonstrated their high reliability. In fact, unexpected failure in these materials results in safety risks, damage and resulting costs.

In order to estimate the expected lifetime of material, component or device it could be necessary to put them in the real operating environment and monitor when

^ Correspondence: valeria.scarano@unifi.it they would die. Being this method not workable because it would cost too much time before the products can be sold, the accelerated life test is proposed to solve this issue. The main goal of lifetime studies is to establish a relationship for the aging process and the stresses causing it, suggest mathematical models, and to verify them. All this is done through an accelerated process, and the results applied to normal operating conditions. Therefore, being able to predict end of life with certain degree of accuracy in a short time span by accelerating the aging process is very useful to design engineers. There are several ways to accelerate the aging process. The most popular are experiments performed at environmental, mechanical and electrical stresses much higher than normal operating conditions. Furthermore the method of stress application (constant or time varying) is fundamental in the life model implementation.

Considering that an important possible application of this material is the soldering in electronic devices, it is necessary to ensure extremely resistant joints, able to sustain the unavoidable electrical, mechanics and environmental stresses that take place during the operating life. Specimens with material were realized for this purpose. Several accelerated models were proposed in literature to obtain lifetime data, considering thermal fatigue, vibration, electrical solicitation or their interaction, for soldering material alloy [3-5], while there is a lack of studies on new ECA accelerated life model, the characterization of their ageing and the relative experimental data analysis [6].

The samples are generally aged for several designated time spans and examined for physical and chemical changes; the scatter in the experimental data from 
any lifetime test advocates the application of statistical methods which are briefly described in the section below. Statistics is frequently used to predict life expectancy. To properly evaluate the data, certain steps are required: Weibull probability distribution and recent contributes are evaluated in order to consider the specific features of lifetime experimental data and modelling [7]. In particular, the structure of the experimental data relating to the statistical modelling approach implies the consideration of random effects and Bayesian methods [8].

\section{Quantitative accelerated testing}

A variety of methods that serve different purposes have been named "accelerated life testing". As we use the term in this reference, accelerated life testing (ALT) involves acceleration of failures with the single purpose of quantification of the ageing of the product also at normal use conditions.

More specifically, accelerated life testing can be divided into two areas: qualitative accelerated testing and quantitative accelerated life testing. In qualitative accelerated testing, the engineer is mostly interested in identifying failures and failure modes without attempting to make any predictions as to the product life under normal use conditions. In quantitative accelerated life testing, the engineer is interested in predicting the life of the product (or, more specifically, life characteristics such as Mean Time to Failure) at normal use conditions, from data obtained in an accelerated life test [9].

Quantitative accelerated life testing consists of tests designed to quantify the life characteristics of the product, component or system under normal use conditions and thereby provide reliability information. Reliability information can include the determination of the probability of failure of the product under use conditions, mean life under use conditions and projected returns and warranty costs. It can also be used to assist in the performance of risk assessments, design comparisons, and so on.

Quantitative accelerated life testing can take the form of usage rate acceleration or overstress acceleration. A simply way to accelerate the life of many products is to run the product more at a higher usage rate. Overstress acceleration, instead, consists of running a product higher than normal levels of some accelerating external stresses to shorten product life or to degrade product performance faster. Typical accelerating stresses are temperature, voltage, mechanical load, thermal cycling, humidity or vibration. Overstress testing is the most common form of accelerated testing.

In order to plan and implement quantitative accelerated life testing we propose the flow chart in Figure 1, in which the identified steps are the following:

1. Choice of failure mechanism to be analyzed.

2. Identification of the stress that activates failure mechanisms and its application mode.

3. Selection of a life distribution.

4. Selection of a life-stress relationship/model.
5. DoE planning.

6. Test implementation.

7. Statistical model: data analysis and parameters estimation.

8. Predicted values of reliability information (in operative conditions).

The proposed procedure it could guarantee an improvement in quality of testing design and implementation. In particular the introduction of the DoE in the accelerated test planning let to consider the fundamental factors and relative levels in order to predict the operating life of device under test in function of the applied severities and so the time to market of the manufacturer. Moreover discover interactions between factors in order to predict the lifetime in accelerated and real condition is today an important challenge to be won.

\section{Electrically conductive adhesive}

In the last years Electrically Conductive Adhesive has increased their importance in electronics because their multiple uses are demonstrating. Some suggested applications are in semiconductor integrated circuit packaging as dieattaching chips to lead-frames; in hybrid micro-electronics as die-attaching of quartz crystal oscillators or as adhesive for electromagnetic interferences and radiofrequency shielding of radiofrequency, microwave and infrared devices. In electronic and opto-electronic circuit assembly the ECA can be used to make electrical contacts in acoustical applications of speakers/microphones, electrical connection of piezoelectric to board, including ink jet heads, MEMS (Micro Electro-Mechanical Systems) and ultrasound devices; for automotive applications in pressure sensing and accelerometer circuits. Moreover ECA could be useful for connections of circuits to $\mathrm{Cu}$ coils in radiofrequency antenna applications such as smart cards and radiofrequency identification tags or for attaching surface mount devices to membrane switch flex circuits. In solarphotovoltaic industry ECA can be used for the electrical connection of transparent conductive oxide (TCO) to printed circuit board (PCB) pads and replacement of solder joints of $\mathrm{Cu} / \mathrm{Sn}$ ribbon wire from cell-to-cell.

In medical applications this material can be useful for circuits requiring implantation or biocompatibility, as electrical connections of die in X-ray circuits, in ultrasound applications $[10,11]$ and in pacemaker. It can attach waveguides, die bonds laser diodes and heatsinks in the high power laser circuits. It can be an electrical connection of ITO (Indium Tin Oxide) to PCBs found in LCD (liquid crystal display) industry or at low temperature for OLED (Organic Light Emitting Diode) displays and organically printable electronics. After the exemplification of several possible future applications of this material it is easy to understand the importance of the study of their performance, trying to discover and then study possible degradation or failure.

Electrically conductive adhesive is a material consisting of epoxy resin filled with metal particles and has 


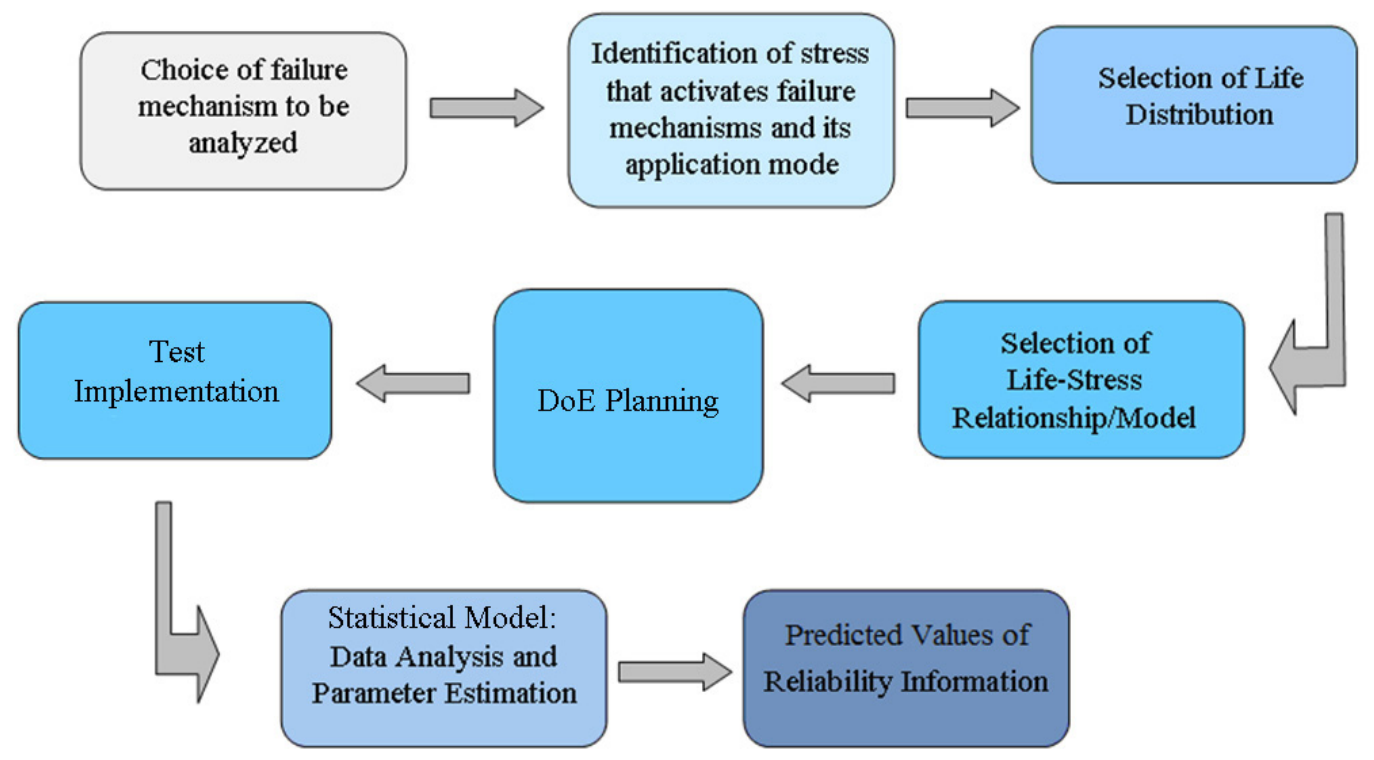

Fig. 1. Accelerated Life Testing flow chart.

two common qualities: it provides a chemical bond between two surfaces and conducts electricity. It has great advantages: it can be used as electrical interconnect on non-solderable substrates such as ceramic or plastics, or to replace solder on thermally sensitive components that cannot withstand $>200{ }^{\circ} \mathrm{C}$ solder processing temperatures [12-14]. Furthermore it is capable of being snap cured in-line, as well as traditional box oven techniques and it appears as a comparable alternative to solder and eutectic die attach, in terms of thermal performance; very commonly no more than $1-2{ }^{\circ} \mathrm{C} /$ Watt difference in thermal resistance. It can be applied in high frequency devices and it is compatible with $\mathrm{Au}, \mathrm{Ag}, \mathrm{Ag}-\mathrm{Pd}$ terminations of surface mount capacitors and resistor. In particular we focused on an isotropic material which is conductive in all directions (Isotropically Conductive Adhesive - ICA). Isotropic conductive materials conduct electricity in all directions equally.

As said above the adhesive part is made up by a matrix of epoxy resin, while the filler particles, which could have different geometric forms, could be Silver, Gold or conductive alloys. For highly conductive adhesives the most popular filler, that creating continuous bridges guides the current inside the material, is Silver (Ag-ICA). This choice is due to its steady and low resistivity $(1.6 \mu \Omega \mathrm{cm}[15])$, its good capability of current carrying and its chemical stability. The negative issue is the possible occurrence of the electromigration phenomenon [16]. The volume fraction of filler, till today to $70-80 \%$, plays a fundamental role in the mechanism of electrical conduction: if high, the probability of a conductive path formation, between the various particles drowned in the matrix of epoxy resin, is greater (conductive chain), on the other side it is also true that at the same time the viscosity of the conductive resin increases making more difficult its use and workability. Therefore, manufacturers of conductive adhesive seek to achieve a compromise between percentage of conduc- tive material and workability request for a versatile use in different electronic, such as soldering. The manufacturers' research focuses particularly on analysis of the network of electrical connections which are formed and are carried out referring to the percolation theory.

Isotropic silver-filled epoxies can be worked at heat or room temperature curing, and can be found in single or two component formulations. Since electrically conductive epoxies require temperatures of only $150{ }^{\circ} \mathrm{C}$ or less to cure, isotropic epoxies are an ideal alternative to solder on thermally sensitive parts. The performance of these materials in particular application was shown in $[10,11]$, instead in order to have a general validity of the research activity we propose specimens were implemented according to a specific geometry and a proper procedure as shown in [17].

In this case-study, a one-component adhesive, Heraeus was examined. It is characterized by a viscosity value equal to $15-25 \mathrm{~Pa} \mathrm{~s}$ (at $50 \mathrm{~s}^{-1}, 23^{\circ} \mathrm{C}$ ).

The necessary prerequisite in order to have a mechanically resistant solder joint using an adhesive polymer is that it has a surface tension less than that of the material of which have to be adhered. If this condition occurs the adhesive is able to effectively wet the surface and then to adhere to it with strength. The epoxides and polyimides have a surface tension lower than that of tin, lead, copper, gold and palladium; then a solder joint with adhesive will have a good mechanical strength if made on these metals or on their alloys. Another problem related to the construction of a solder joint with adhesive is that small molecules of contaminants can penetrate into the matrix of the polymer [18]. In particular this is the case for the molecules of water, which once penetrated in the glue, can reach on the metal surface on which insists the soldering and create oxide layers, leading to an increase of the electrical resistance [19].

It is important to consider that the effect of curing on the soldering reliability is relevant only if a minimum 
level of polymerization is not reached. In fact, if the curing has already produced the polymerization of $70-80 \%$ of the material, a continuation of the curing does not produce significant advantages in terms of reliability. On the contrary, if the polymerization does not reach the degree indicated, the mechanical fragility increases such as the risk that water molecules penetrate the polymer and oxidize the soldering surface.

Form the mechanical resistance point of view the conductive adhesives were described as poor in the event of impact and shock, but with the improvement of the chemical formulations of the polymers, this problem has been limited by inserting elastomers in the compound or, in general, by improving the adhesion strength and the damping capacity of the effort within the polymeric material.

The aim of this research activity is to study the reliability of conductive adhesive specimens, in particular Heraeus PC3000, by means of accelerated tests in temperature in order to identify specific failure mechanisms and to evaluate the useful life.

Given the peculiarities of the conductive adhesives, failure mechanisms that occur could be very different from those which characterize the metal soldering where the formation of intermetallic compounds and the enlargement of the grains are the main factors that can lead to failure. In the case of adhesives, the failure mechanisms can be traced mainly to two cases: the formation of an insulating layer (typically an oxide) at the interface or the fracture which generates a loss of metal contact that may be due to a decrease of the strength of adhesion, to a relaxation of the compressive strength or to thermal or mechanical deformation.

The compressive strength that allows maintaining the contact between the filler particles, and thus ensures the electrical connection, is obtained thanks to the narrowing of the adhesive matrix during the curing phase of the adhesive. Both the internal cohesion strength of the adhesive matrix and the strength of adhesion at the interface should be such as to maintain the necessary compressive strength, which, however, tends to be affected by thermal expansion of the adhesive, by the moisture absorption and also by mechanical stress.

The loss of adhesion, with consequent delamination and loss of mechanical strength can occur both within the adhesive material and the interface bonding. The delamination can be immediately complete, with a full detachment, or also be partial, with the formation of fractures which could lead to full detachment with the progress of time.

The factors that can lead to delamination are mainly due to:

- an insufficient progress of the polymerization process that can be caused by not adequate time and/or temperature;

- a mix of the adhesive components in an incorrect stechiometric ratio;

- an absent or inadequate pre-treatment of the attachment surface;
- a high difference between the coefficients of thermal expansion of the glued parts and the adhesive;

- moisture absorption or entrapment of other impurities.

\section{Experimental data and statistical models}

In this paper, experimental and statistical approach is carried out in order to study product lifetime and, in particular, ECA lifetime. More precisely, the first step of the analysis should consider the planning of trials by evaluating the specific features and characteristics of the involved ECAs and, in addition, the environmental and noises factors which can be included in lifetime experiments to stress the ECAs performances. Secondly, specific statistical models for data analysis are suggested.

\subsection{Planning of the experimental design}

The design of experiment is a wide and fundamental methodology of the statistics theory; at the beginning, the classical experimental design was mainly directed to study specific problems sourcing in agriculture; therefore, some basic and classical definitions, like the definition of blocks and sub-experimental factors or Latin-square designs, were related to this specific kind of applications [20]. Nevertheless, since 1950s, many theoretical developments have been formulated in order to extend the basic and simple classical designs and linear modelling; furthermore, these new theoretical issues have been differently developed by considering the specific fields of application. Optimal design criteria and Response Surface Methodology (RSM) date back to this period.

A great impulse to the evolution of experiments theory was further originated by the technological field and the concept of quality improvement. The study of quality products or processes involving the design of experiments dated back since 1940s and the definition of the fractional factorial design together with RSM has been largely developed especially in 1980s and 1990s; the robust design approach through RSM $[21,22]$ or Generalized Linear Models (GLMs) [23-25] gave a notably boost to the use and application of design of experiments in new fields.

In this context and more recently, reliability statistical analysis has requested a systematic collection of data which has been naturally lead to the use of the experimental design theory [26]. Undoubtedly, reliability analysis is a great challenge for experiments relating to specific issues, such as the structure of data and the corresponding non-linear statistical models (Sect. 4.2).

In our case study, the planning of the experimental design has been built starting from the experimental design planned for the ECA's optimization process [17]. Furthermore, we may ideally divide the experimental programming in two steps: (i) the first step relates to the planning and optimization in order to set an optimal solution for ECA following the robust design concepts; (ii) the second step uses the experimental trials carried out at the first step. 
By considering this second step, each ECA is separately planned through the 18 runs of the first step; each run is stressed by Thermal Cycling Test (TCT). We decided to plan Thermal Cycling Test in order to induce crack and so resistivity variation in specimens and then to implement ECA solder joint reliability. This experimental approach will be used to study the effects of maximum temperature, ramp rates, dwell times, which are temperature profile (trapeze-like cycle) on ECA solder joint life. A detailed study of maximum temperature, dwell time and ramp rate effects will carried out and we hypothesize that the study of the possible effects will show how accelerated thermal cycling profiles can be optimized to improve test efficiency. ECA life could vary with the selected factors because damage in the ramp is affected by strain rate, temperatures and times within ramp. Furthermore, damage in dwell is affected by preceding strain rate, temperature and time within dwell.

In this work, the sensitivity to maximum temperature and temperature range will be studied. This experimental study evaluates the maximum temperature and, consequently, the temperature range variation. Note that the considered temperature range could vary from $100{ }^{\circ} \mathrm{C}$ to $150{ }^{\circ} \mathrm{C}$. Intuitively, we expect that cycles to failure progressively decreases with the increase in temperature range, consequently with the achievement of maximum temperature. We decided to fix at $25{ }^{\circ} \mathrm{C}$ the minimum temperature profile, that will be the reference temperature at which the electrical performance of the tested material will be measured.

The ramp rate is characterized by a nominal value of $1{ }^{\circ} \mathrm{C} \mathrm{min}^{-1}$. The studied ramp rates reach as maximum value $5{ }^{\circ} \mathrm{C} \mathrm{min}^{-1}$. Higher ramp rates are not considered as they may lead to interaction from mechanisms other than those considered in this paper. This study is of particular interest since no definitive experimental results are available in the literature on the effect of thermal ramp rate on cycles to failure of ECA.

The dwell time for the nominal value is $10 \mathrm{~min}$. The other investigated dwell time values achieve the maximum value to $30 \mathrm{~min}$, considering that the increasing dwell time at high temperature decreases the life, but with decreasing sensitivity when dwell time increases [27].

We expect that life will have dependence from the selected factors, it will suggest sensitivity to different material properties in ramp and dwell. In fact, we suppose that there will be dependencies and potential interactions to address through the implemented statistical methods.

The choice of level factors allows us to increase the stress severity in order to induce acceleration in material degradation. As final aim it will be important to extrapolate the acceleration factor and its sensitivity to the other factors and corresponding levels. After the Accelerated TCT, it will be interesting to evaluate the coefficient of thermal expansion on the tested ECA in order to understand the predicted use of these new lead-free materials in electronic devices.

It must be noted that the response variable is the electrical resistance in both experimental steps, however, in
Table 1. Factors for the design of experiment.

\begin{tabular}{ccc}
\hline Factors & Low level & High level \\
\hline Dwell temperature & $130{ }^{\circ} \mathrm{C}$ & $170{ }^{\circ} \mathrm{C}$ \\
Ramp rate & $1{ }^{\circ} \mathrm{C} \mathrm{min}$ min $^{-1}$ & $3{ }^{\circ} \mathrm{C} \mathrm{min}$ min $^{-1}$ \\
Dwell time & $10 \mathrm{~min}$ & $30 \mathrm{~min}$ \\
\hline
\end{tabular}

the second experimental step we have multiple electrical resistance response variables, each collected at a specific value of thermal cycle $t(t=1, \ldots T)$. Thus, $Y_{\text {it }}$ is the electrical response variable related to the $i$ th trial collected at cycle $t$.

We suppose that these $T$ response variables will be strongly correlated and, obviously, we do not know a-priori the amount of failures at cycle $t$.

Two relevant issues should be evaluated during the experimental planning and subsequent analysis:

(1) for each specimen, a random allocation is performed between the two experimental steps; thus, a random effect will be evaluated in the statistical model;

(2) at least two specimens are considered for each trial of the new second experimental design.

Therefore, the final experimental design for the second step is a complete factorial design with three factors at two levels and a centre point replication. Through this experimental design it is possible to estimate the three first order interactions, even though only two specimens are analysed for each new trial. In order to combine 18 specimens and 9 experimental trials, a program of random number generation has been used, not to influence with personal choice the combination.

As above the conductive adhesives can be used in a wide range of applications and environments, therefore it appears necessary to design test in order to have general validity. In this research activity used the model of accelerated test based on cycles of temperature.

To produce fatigue failures or fracture, the studied temperature cycles were designed by combining the factors and levels reported in Table 1. Eight profiles were obtained plus one in the center of the experimental design; the obtained testing profiles are summarized in Figure 2.

The minimum temperature was $25{ }^{\circ} \mathrm{C}$ (typically corresponding to the ambient temperature), reference temperature for the parameters control. In this first phase of experimentation the relative humidity was not considered as environmental stress in order to characterize the conductive adhesive to single stress with the aim to avoid the occurrence of faults different from those we wanted to investigate. During the test the severities were monitored in order to control possible differences between the ideal and the real thermal profile.

To monitor the behaviour of the specimens before and after the test, we consider the electrical resistance as the parameter chosen to monitor the degradation of the materials.

In this first phase the experimentation was performed with the more severe test and the less severe, so the testing profile $1\left(+25{ }^{\circ} \mathrm{C} \div+170{ }^{\circ} \mathrm{C}, 3{ }^{\circ} \mathrm{C} \mathrm{min}-1,30 \mathrm{~min}^{-1}\right)$ was 


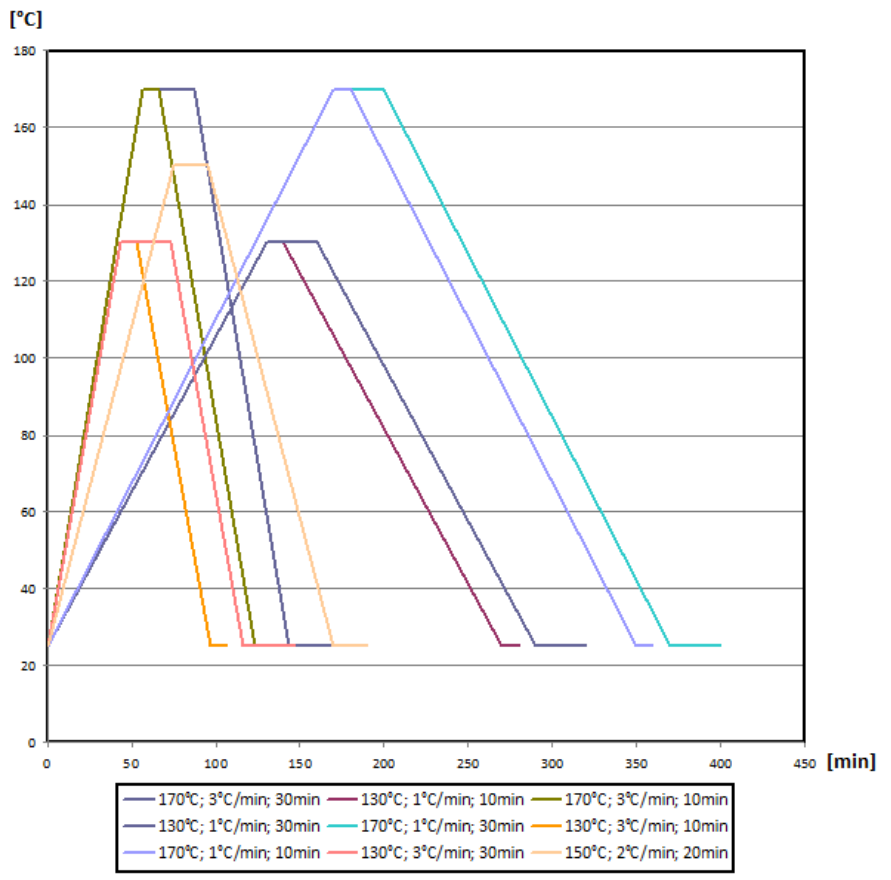

Fig. 2. Plots of testing profiles.

Table 2. Testing profile $1\left(170{ }^{\circ} \mathrm{C}, 3{ }^{\circ} \mathrm{C} \min ^{-1}, 30 \mathrm{~min}\right)$.

\begin{tabular}{cc}
\hline \multicolumn{2}{c}{$170{ }^{\circ} \mathrm{C} ; 3{ }^{\circ} \mathrm{C} / \mathrm{min} ; 30 \mathrm{~min}$} \\
\hline Minimum temperature & $25^{\circ} \mathrm{C}$ \\
Maximum temperature & $170{ }^{\circ} \mathrm{C}$ \\
Ramp rate & $3{ }^{\circ} \mathrm{C} \mathrm{min}{ }^{-1}$ \\
Upper e lower Dwell time & $30 \mathrm{~min}$ \\
Total cycle duration & $173.33 \mathrm{~min}$ \\
\hline
\end{tabular}

Table 3. Testing profile $2\left(130{ }^{\circ} \mathrm{C}, 1{ }^{\circ} \mathrm{C} \min ^{-1}, 10 \mathrm{~min}\right)$.

\begin{tabular}{cc}
\hline \multicolumn{2}{c}{$130{ }^{\circ} \mathrm{C} ; 1{ }^{\circ} \mathrm{C} \mathrm{min}{ }^{-1} ; 10 \mathrm{~min}$} \\
\hline Minimum temperature & $25^{\circ} \mathrm{C}$ \\
Maximum temperature & $130{ }^{\circ} \mathrm{C}$ \\
Ramp rate & $1{ }^{\circ} \mathrm{C} \mathrm{min}$ \\
Upper e lower Dwell time & $10 \mathrm{~min}$ \\
Total cycle duration & $280.00 \mathrm{~min}$ \\
\hline
\end{tabular}

applied to the samples named N01 and N016 and the testing profile $2\left(+25^{\circ} \mathrm{C} \div+130{ }^{\circ} \mathrm{C}, 1^{\circ} \mathrm{C} \mathrm{min}{ }^{-1}, 10 \mathrm{~min}\right)$ to specimens N13 and N18. All the values necessary for the proper cycles design, as minimum temperature, maximum temperature, upper and lower dwell time, ramp rate and total duration of each cycle in the Tables 2 and 3 were summarized, and the relative plot shown in Figures 3 and 4.

After the configuration of the test, the samples were located in the centre of the chamber, and the temperature in this area was monitored with Pt100 during the tests. The specimens were bound in order to avoid those vibrations, caused by the normal operation of the chamber compressors, could move samples from their position (see Fig. 5). The same configuration has been maintained for the recurrence of all cycles.

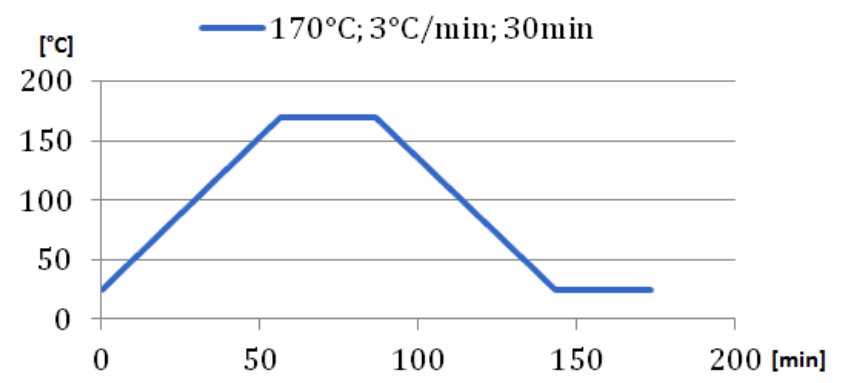

Fig. 3. Plot of testing profile 1.

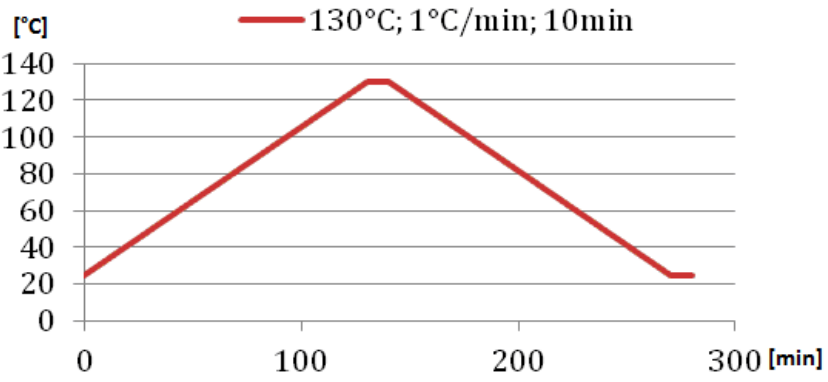

Fig. 4. Plot of testing profile 2 .

Chosen the measure of electrical resistance for the verification or not of a fault occurrence, the measurement was implemented by means of a Hewlett Packard 34401A multimeter, in 4-wire mode.

Before subjecting specimens to reliability tests were characterized by initial resistance measurements. Intermediate measurements were implemented to evaluate the possible variation of resistance; the measurement is repeated at the end of each 10 cycles. The measurements plots are shown in Figure 6.

Resistance measurements carried out during the test and at the end of the test any failure mechanism did not reveal the occurrence, conversely it has been detected a resistance values lower than that obtained from measurements before the testing start.

The aim of this work was to verify the occurrence of a fault caused by an increase, at least of $20 \%$, of the specimens' resistance. As already mentioned, the final resistance measurements have not deviated excessively compared to those done before test, in particular in Figure 6 it is possible to see a decrease of the resistance, which does not exceed the value of $30 \%$ for the four specimens, both for N1 and N16 subject to the accelerated profile 1 and for N13 and N18 subject to the accelerated profile 2. The average decrease is equal to $22 \%$, so it is clear that no faults are occurred.

Also from the visual inspection the samples are not altered, except for the surface substrate of samples N01 and N16 which is blackened but not fractured, undoubtedly due to the high temperatures $\left(170^{\circ} \mathrm{C}\right)$ reached under test. The decrease of observed electrical resistance could be attributable to the continuation of the curing phase, 


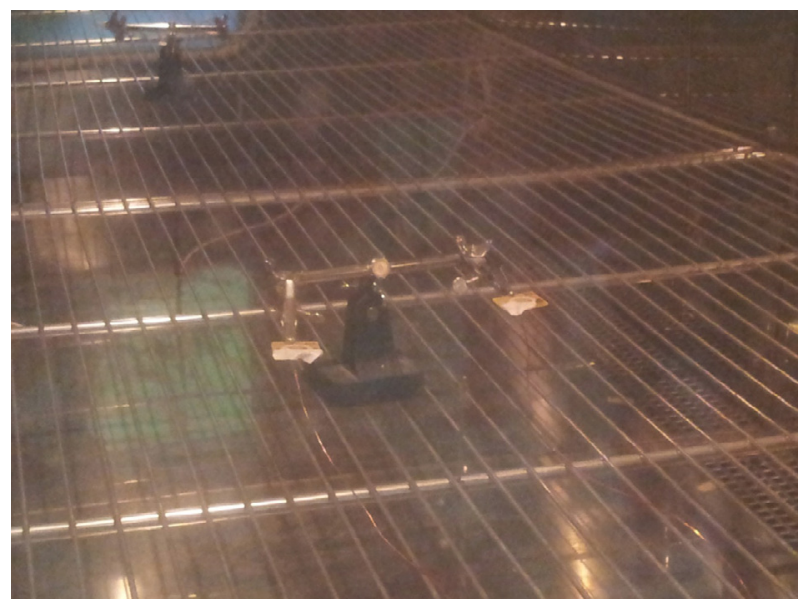

Fig. 5. Specimens under test.

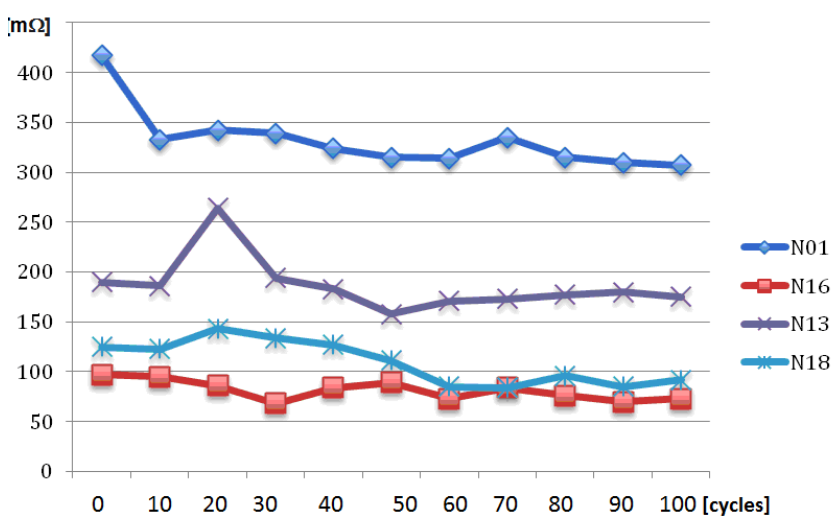

Fig. 6. Plot of the electrical resistance measurement $(\mathrm{m} \Omega)$.

this behaviour of the material does not affect the operation of the devices on which it could be applied.

\subsection{The statistical model}

In this regard, specific statistical models should be applied in order to consider the aforementioned situation and, eventually, non-linear effects. Particularly, in the recent literature, extensions to previous statistical reliability models are introduced to treat experiments not coming from completely randomized design (CRD) [8] together with non-fixed effects [7]. Thus, Weibull non-linear mixed models can be applied to evaluate blocks and random effects.

The two-parameter Weibull distribution has the following Probability Distribution Function (PDF) for $N$ statistical units $(i=1, \ldots N)$ :

$$
f\left(t_{i}\right)=\frac{\beta}{\eta}\left(\frac{t_{i}}{\eta}\right)^{\beta-1} e^{-\left(\frac{t_{i}}{\eta}\right)^{\beta}}
$$

where $\beta>0$ and $\eta>0$ are the shape and the scale parameters, respectively.

The corresponding likelihood function is:

$$
l(\beta, \eta)=\prod_{i} f\left(t_{i}\right)
$$

It must be noted that this expressions are related to a life test data for a CRD according to [28].

Furthermore, when considering the Weibull distribution, formula (1), and reliability statistical data, a further issue should be taken into account.

In fact, in this case, the specific structure of data is strictly tied to the estimation of the corresponding statistical model: often, experimental units do not have the same failure time. Thus, censored data are generated and specific type of censoring may be defined. For example, two common types of right censored data are Type I and Type II, related to time censored and failure censored, respectively. When considering these two types of censored data, the likelihood becomes:

$$
l(\beta, \eta)=C \prod_{i}\left[f\left(t_{i}\right)^{\delta_{i}}\left[1-F\left(t_{i}\right)\right]^{1-\delta_{i}}\right.
$$

where $\delta_{i}$ is a dummy variable specified for each statistical unit and it is equal to zero if the unit is right censored and equal to one if observed; $F\left(t_{i}\right)$ is the cumulative distribution function, while $C$ is a constant which varies according to the kind of censoring.

It must be noted that this section is included in order to present the statistical models for reliability useful for this study; nevertheless this part may not cover the methodological issues that could arise during the statistical analysis, after the completion of the experimental step.

\section{Conclusion}

The relevance of the accelerated stress test on lead-free materials for electronics is focused in the paper. Being fundamental in a wide range of applications the characterization of such new materials from the reliability point of view, a procedure to design the accelerated test by means of Design of Experiment technique has been proposed. The approach let to guarantee an improvement in quality of testing design and evaluation of results. Considering all factors with the corresponding levels for the selected environmental stress (particular attention is voted to the temperature), the introduction of the DoE let to correlate the fundamental factors and levels with predicted operating life of the device under test.

The first experimentation was used to better understand the response of ECA solder material under thermal cycling conditions. The statistical approach, which has also been validated for maximum temperature, dwell time and thermal rate effects, can be used to optimize accelerated test conditions. This experimental study, from the point of view of test engineers, brings to the optimization of maximum temperature, ramp rate and dwell times for best test-time compression. In industrial world solder interconnects or electronic packages are typically tested under accelerated laboratory conditions, so the test time compression is important also from an economic point of view. The most significant contribution of this study is a rigorous approach to the implementation of ECA reliability test to predict durability based on cyclic test; after 
implemented cycles the results proved that the electrical performance for all the specimens.

The suggested approach, a challenge by the research point of view, will be interesting also by industrial world because manufacturers will to design accelerated life test in function of the time to market of their products.

Acknowledgements. Our thanks to Eng. Niccolò Pierossi for his support during first phase of experimental testing.

\section{References}

1. W. Li, R.C. Feng, Highly Accelerated Life Test for the Reliability Assessment of the Lead-Free SMT Mainboard, Int. Microsystems Pack, Assembly Conf. (2006), pp. 1-4

2. V. Venkatadri, Y. Liang, X. Yan, E. Cotts, K. Srihari, P. Borgesen, Accelerating the effects of aging on the reliability of lead free solder joints in a quantitative fashion, Electronic Comp. Technol. Conf. (2009), pp. 398-405

3. F.X. Che, H.L.J. Pang, F.L. Wong, G.H. Lim, T.H. Low, Vibration fatigue test and analysis for flip chip solder joints, Electronics Pack. Technol. Conf. (2003), pp. 107113

4. T. Eckert, W.H. Muller, N.F. Nissen, H. Reichl, A Solder Joint Fatigue Life Model for Combined Vibration and Temperature Environments, Electronic Comp. Technol. Conf. (2009), pp. 522-528

5. H. Qi, M. Osterman, M. Pecht, Modeling of Combined Temperature Cycling and Vibration Loading on PBGA Solder Joints Using an Incremental Damage Superposition Approach, IEEE Trans. Adv. Pack. 31, 463-472 (2008)

6. Y. Li, C.P. Wong, Recent advances of conductive adhesives as a lead-free alternative in electronic packaging: Materials, processing, reliability and applications, Mater. Sci. Eng. 51, 1-35 (2006)

7. R.V. Leon, R. Ramachandran, A.J. Ashby, J. Thyagarajan, Bayesian modelling of accelerated life tests with random effects, J. Qual. Technol. 39, 1-14 (2006)

8. L.J. Freeman, G.G. Vining, Reliability data analysis for life tests experiments with subsampling, J. Qual. Technol. 42, 233-241 (2010)

9. W.B. Nelson, Accelerated Testing: Statistical Models, Test Plans, and Data Analysis (Wiley-Interscience Paperback Series, NJ, 2004)

10. M. Catelani, V.L. Scarano, F. Bertocci, Implementation and Characterization of a Medical Ultrasound Phased Array Probe with New Pb-free Soldering Materials, IEEE Trans. Instrum. Meas. 59, 2522-2529 (2010)

11. M. Catelani, V.L. Scarano, F. Bertocci, Experimental Stress Characterization of a Biomedical Ultrasound Probe Soldered With Innovative Silver Isotropically Conductive Adhesive, IEEE Trans. Instrum. Meas. 61, 719-728 (2012)
12. Y. Tao, Y. Xia, H. Wang, F. Gong, H. Wu, G. Tao, Novel isotropical conductive adhesives for electronic packaging application, IEEE Trans. Adv. Pack. 32, 589-592 (2009)

13. D. Shangguan, Lead-Free Solder Interconnect Reliability (ASM international, Materials Park, Ohio, 2005)

14. I. Mir, D. Kumar, Recent advances in isotropic conductive adhesives for electronics packaging applications, Int. J. Adhes. Adhes. 28, 362-371 (2008)

15. D. Klosterman, L. Li, J.E. Morris, Materials Characterization, Conduction Development, and Curing Effects on Reliability of Isotropically Conductive Adhesives, IEEE Trans. Comp. Pack. Manufact. Technol., Part A 21, 23-31 (1998)

16. D.D. Chang, P.A. Crawford, J.A. Fulton, R. McBride, M.B. Schmidt, R.E. Sinitski, C.P. Wong, An Overview and Evaluation of Anisotropically Conductive Adhesive Films for Fine Pitch Electronic Assembly, IEEE Trans. Comp. Hybr. Manuf. Technol. 16, 828-835 (1993)

17. M. Catelani, V.L. Scarano, F. Bertocci, R. Berni, Optimization of the soldering process with ECAs in electronic equipment: characterization measurement and experimental design, IEEE Trans. Comp. Pack. Manuf. Technol. 1, 1616-1626 (2011)

18. L. Li, J.E. Morris, J. Liu, Z. Lai, L. Ljungkrona, C. $\mathrm{Li}$, Reliability and failure mechanism of isotropically conductive adhesives joints, Electronic Comp. Technol. Conf. (1995), pp. 114-120

19. Q.K. Tong, D.L. Markley, G. Frederickson, R. Kuder, D. $\mathrm{Lu}$, Conductive Adhesives with Stable Contact Resistance and Superior Impact Performance, Electronic Comp. Technol. Conf. (1999), pp. 347-352

20. W.G. Cochran, G.M. Cox, Experimental Designs, 2nd edn. (John Wiley, New York, 1957)

21. R.H. Myers, A.I. Khuri, G.G. Vining, Response Surface alternatives to the Taguchi robust parameter design approach, Am. Stat. 46, 131-139 (1992)

22. G.G. Vining, R.H. Myers, Combining Taguchi and response surface philosophies: a dual response approach, J. Qual. Technol. 22, 38-45 (1990)

23. J.A. Nelder, Y. Lee, Generalized Linear Models for the analysis of Taguchi-type experiments, Appl. Stoch. Model D. A. 7, 107-120 (1991)

24. Y. Lee, J.A. Nelder, Robust design via Generalized Linear Models, J. Qual. Technol. 35, 2-12 (2003)

25. A.H. Dror, D.M. Steinberg, Robust design for Generalized Linear Models, Technometrics 48, 520-529 (2006)

26. L.W. Condra, Reliability Improvement With Design of Experiments, Quality and Reliability, 2nd edn. (Marcel Dekker, Inc., New York, 2001)

27. P. Sharma, A. Dasgupta, Micro-mechanics of creep-fatigue damage in PB-SN solder due to thermal cycling - Part II: mechanistic insights and cyclic durability predictions from monotonic data, ASME Trans. J. Electron. 124, 298-304 (2002)

28. J.F. Lawless, Statistical Models and Methods for Lifetime Data (John Wiley \& Sons, Hoboken, NJ, 2003) 